\title{
Determinants of suboptimal breast-feeding practices in Pakistan
}

\author{
Tabish Hazir ${ }^{1, *}$, Dure-Samin Akram², Yasir Bin Nisar ${ }^{3}$, Narjis Kazmi $^{1}$, \\ Kingsley E Agho ${ }^{4}$, Saleem Abbasi ${ }^{1}$, Amira M Khan ${ }^{1}$ and Michael J Dibley ${ }^{5}$ \\ 'ARI Research Cell, Children's Hospital, Pakistan Institute of Medical Sciences, Sector: G-8/3, Islamabad \\ 44000, Pakistan: ${ }^{2}$ Health Education and Literacy Program, Sindh, Pakistan: ${ }^{3}$ Population Council, Islamabad, \\ Pakistan: ${ }^{4}$ School of Medicine, University of Western Sydney, Penrith, New South Wales, Australia: ${ }^{5}$ Sydney \\ School of Public Health, Sydney Medical School, University of Sydney, Sydney, New South Wales, Australia
}

Submitted 16 July 2011: Final revision received 4 May 2012: Accepted 11 May 2012: First published online 4 July 2012

\begin{abstract}
Objective: Exclusive breast-feeding is estimated to reduce infant mortality in lowincome countries by up to $13 \%$. The aim of the present study was to determine the risk factors associated with suboptimal breast-feeding practices in Pakistan. Design: A cross-sectional study using data extracted from the multistage cluster sample survey of the Pakistan Demographic and Health Survey 2006-2007. Setting: A nationally representative sample of households. Subjects: Last-born alive children aged 0-23 months (total weighted sample size 3103).

Results: The prevalences of timely initiation of breast-feeding, bottle-feeding in children aged 0-23 months, exclusive breast-feeding and predominant breastfeeding in infants aged $0-5$ months were $27 \cdot 3 \%, 32 \cdot 1 \%, 37 \cdot 1 \%$ and $18 \cdot 7 \%$, respectively. Multivariate analysis indicated that working mothers $(\mathrm{OR}=1 \cdot 48$, $95 \%$ CI $1 \cdot 16,1 \cdot 87 ; P=0 \cdot 001)$ and mothers who delivered by Caesarean section (OR $=1 \cdot 95,95 \%$ CI $1 \cdot 30,2 \cdot 90 ; P=0 \cdot 001)$ had significantly higher odds for no timely initiation of breast-feeding. Mothers from North West Frontier Province were significantly less likely (OR $=0.37,95 \%$ CI $0 \cdot 23,0 \cdot 59 ; P<0 \cdot 001$ ) not to breast-feed their babies exclusively. Mothers delivered by traditional birth attendants had significantly higher odds to predominantly breast-feed their babies $(\mathrm{OR}=1 \cdot 96,95 \% \mathrm{CI} 1 \cdot 18,3 \cdot 24 ; P=0 \cdot 009)$. The odds of being bottle-fed was significantly higher in infants whose mothers had four or more antenatal clinic visits $(\mathrm{OR}=1 \cdot 93,95 \% \mathrm{CI} 1 \cdot 46,2 \cdot 55 ; P<0 \cdot 001)$ and belonged to the richest wealth quintile $(\mathrm{OR}=2 \cdot 41,95 \%$ CI $1 \cdot 62,3 \cdot 58 ; P<0 \cdot 001)$.

Conclusions: The majority of Pakistani mothers have suboptimal breast-feeding practices. To gain the full benefits of breast-feeding for child health and nutrition, there is an urgent need to develop interventions to improve the rates of exclusive breast-feeding.
\end{abstract}

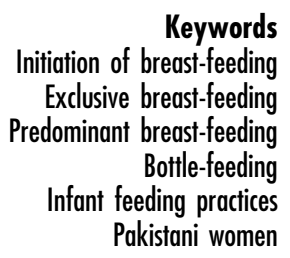

The advantages of exclusive breast-feeding (EBF) and the risks of higher morbidity and mortality associated with suboptimum feeding practices in children are well documented $^{(1-6)}$. For this reason, the WHO infant feeding guidelines recommend that newborns should be put to the breast within the first hour of birth (timely initiation of breast-feeding) and infants should be exclusively breast-fed for the first 6 months of life and introduced to nutritionally adequate and safe complementary foods thereafter, with breast-feeding continuing up to 2 years of age to achieve optimal growth, development and health $^{(7)}$. A recent analysis shows that suboptimum breast-feeding, especially non-EBF in the first 6 months of life, results in 1.4 million deaths annually and $10 \%$ of the disease burden in children $<5$ years of age ${ }^{(8)}$. It is estimated that $10-15 \%$ of deaths in children aged $<5$ years in-resource poor countries could be prevented through achievement of $90 \%$ coverage with EBF alone ${ }^{(9)}$. Predominant breast-feeding (PBF) is defined as feeding plain water or water-based drinks and fruit juices in addition to breast milk in infants $<6$ months of age ${ }^{(10)}$. Morbidity is increased significantly in predominantly breast-fed babies, especially from gastroenteritis and respiratory tract infections ${ }^{(8,11)}$. Likewise, bottle-feeding has been recognized as detrimental to the health and intellectual development of children ${ }^{(1,12)}$.

Pakistan is the sixth most populous country in the world and the second most populated country in the South Asian region. The level of socio-economic development is still low and a quarter of the entire population lives below the 
poverty line ${ }^{(13)}$. The health profile of Pakistan is characterized by a high population growth rate, high infant and child mortality rates, a high maternal mortality ratio and a high burden of communicable diseases in children ${ }^{(14)}$. Pakistan would gain enormous health and economic benefits by improving infant feeding practices. Identification and recognition of the barriers to EBF in communities is essential for developing effective nutrition education and behaviour change strategies targeting families at heightened risk of suboptimal infant feeding behaviours. The purpose of the present study was to determine the factors associated with non-initiation of timely breast-feeding, non-EBF, PBF and bottle-feeding after controlling for individual-, household- and community-level characteristics.

\section{Methodology}

\section{Data source}

We analysed data from the 2006-2007 Pakistan Demographic and Health Survey (PDHS), which was carried out by the National Institute of Population Studies and Macro International. The 2006-2007 PDHS used a stratified, multistage cluster sampling strategy with urban and rural samples drawn separately and in proportion to the population of each province. Geographic sampling units were obtained for both urban and rural areas and random household sampling was conducted to select units. A total of 10023 ever-married women were interviewed with a response rate of $94.5 \%$. A detailed birth and feeding history for the last 5 years preceding the survey was asked from all of the ever-married women interviewed ${ }^{(14)}$.

The data set was downloaded from the public access website (http://www.measuredhs.com). Afterwards, it was inspected for data quality, completeness of information and comparability of variables required for the present analysis. The required variables from the data set were selected and the files were constructed. We selected lastborn children aged $<24$ months at the time of the survey and living with respondents/mothers. The total weighted number of children aged 0-23 months was 3103 .

\section{Measures}

The dependent variables in the present study were the timely initiation of breast-feeding (0-23 months) and rates of $\mathrm{EBF}$ ( $<6$ months), PBF ( $<6$ months) and bottle-feeding ( $0-23$ months). Indicators were estimated according to the key factors described by the WHO in $2008^{(15)}$. A household wealth index was constructed from data collected in the household questionnaire, using methods recommended by the World Bank Poverty Network and $\mathrm{UNICEF}^{(16)}$, and was divided into five equal categories to classify the households by economic status.

The explanatory variables were classified into three levels, i.e. individual, household and community. The individual level attributes included age, sex, birth weight of the child, mother's age, working status of the mother in the past 12 months, highest education level achieved and marital status at the time of interview. Information on the number of antenatal clinic (ANC) visits, place of delivery, mode of delivery, type of delivery assistance availed, birth order and postnatal contacts (PNC) with a health-care provider was also obtained. Household wealth index was included as a household-level variable. Community-level attributes specific to breast-feeding such as policies and practices that support breast-feeding, implementation of the marketing code for breast milk substitutes and cultural norms regarding breast-feeding were not available in the current DHS data set. Therefore, only the geographical region (province) and residential area (urban and rural) were considered.

\section{Statistical analysis}

The EBF indicator was expressed as a dichotomous variable with category 0 for EBF and category 1 for non-EBF; timely initiation of breast-feeding as category 0 for timely initiation and category 1 for no timely initiation; the PBF indicator was expressed as category 0 for non-PBF (EBF or bottle-feeding in infants $<6$ months separately) and category 1 for PBF; while the bottle-feeding indicator was expressed as category 1 for bottle-feeding and category 0 for not bottle-feeding. These variables were examined against a set of independent variables (individual, household and community characteristics) in order to determine the prevalence of timely initiation of breast-feeding, EBF, $\mathrm{PBF}$ and bottle-feeding and factors associated with no timely initiation of breast-feeding, non-EBF, PBF and bottle-feeding. Analyses were performed using the STATA statistical software package version 10 (StataCorp, College Station, TX, USA) with 'svy' commands to allow for adjustments for the cluster sampling design used in the surveys when estimating confidence intervals around prevalence estimates. The $\chi^{2}$ test was used to test the significance of associations.

Associations were examined by univariate analyses (unadjusted OR and 95\% CI) and then by multivariate logistic regression (adjusted OR (AOR) and 95\% CI) analysis. In the univariate analysis, OR and 95\% CI were calculated in order to assess the unadjusted risk of independent variables on feeding practices. Multivariate logistic regression was employed to estimate the OR adjusted for the independent variables. A stepwise backwards elimination approach was used for model construction, retaining those variables with $P<0.05$ in the final model.

\section{Results}

In the current analysis, 3103 (weighted total) children aged 0-23 months were selected. Table 1 lists the individual-, household- and community-level characteristics of these children. Almost a quarter of the mothers $(22 \cdot 5 \%)$ had 
Table 1 Individual-, household- and community-level characteristics of children aged 0-23 months ( $n$ 3103); secondary analysis of data from the Pakistan Demographic and Health Survey (PDHS) 2006-2007

\begin{tabular}{|c|c|c|}
\hline Characteristic & $n$ & $\%$ \\
\hline \multicolumn{3}{|l|}{ Individual-level factors } \\
\hline \multicolumn{3}{|l|}{ Maternal working status ( $n$ 3099) } \\
\hline Non-working & 2403 & $77 \cdot 5$ \\
\hline Working (past 12 months) & 696 & $22 \cdot 5$ \\
\hline \multicolumn{3}{|l|}{ Maternal education } \\
\hline No education & 1981 & $63 \cdot 8$ \\
\hline Primary & 474 & $15 \cdot 3$ \\
\hline Secondary and above & 648 & $20 \cdot 9$ \\
\hline \multicolumn{3}{|l|}{ Father's education ( $n$ 3096) } \\
\hline No education & 1074 & $34 \cdot 7$ \\
\hline Primary & 525 & $17 \cdot 0$ \\
\hline Secondary and above & 1497 & $48 \cdot 3$ \\
\hline \multicolumn{3}{|l|}{ Literacy ( $n$ 3095) } \\
\hline Cannot read at all & 1945 & $62 \cdot 8$ \\
\hline Able to read only passage & 1150 & $37 \cdot 2$ \\
\hline \multicolumn{3}{|l|}{ Mother's age (years) } \\
\hline $15-19$ & 172 & $5 \cdot 5$ \\
\hline 20-34 & 2409 & $77 \cdot 6$ \\
\hline $35-49$ & 522 & $16 \cdot 8$ \\
\hline \multicolumn{3}{|l|}{ Marital status } \\
\hline Currently married & 3081 & $99 \cdot 3$ \\
\hline Formerly married* & 22 & 0.7 \\
\hline \multicolumn{3}{|l|}{ Birth order } \\
\hline First-born & 609 & $19 \cdot 6$ \\
\hline Second- to fourth-born & 1522 & $49 \cdot 1$ \\
\hline Fifth-born or higher & 971 & $31 \cdot 3$ \\
\hline \multicolumn{3}{|l|}{ Preceding birth interval ( $n$ 3094) } \\
\hline No previous birth & 609 & $19 \cdot 7$ \\
\hline $0-14$ months & 166 & $5 \cdot 4$ \\
\hline 14-24 months & 724 & $23 \cdot 4$ \\
\hline$\geq 25$ months & 1595 & $51 \cdot 5$ \\
\hline \multicolumn{3}{|l|}{ Sex of baby } \\
\hline Male & 1644 & $53 \cdot 0$ \\
\hline Female & 1459 & $47 \cdot 0$ \\
\hline \multicolumn{3}{|l|}{ Age of child (months) } \\
\hline $0-5$ & 955 & $30 \cdot 8$ \\
\hline $6-11$ & 811 & $26 \cdot 1$ \\
\hline $12-17$ & 843 & $27 \cdot 2$ \\
\hline $18-23$ & 494 & $15 \cdot 9$ \\
\hline \multicolumn{3}{|l|}{ Perceived birth size of baby $(n 3100)$} \\
\hline Small & 1056 & $34 \cdot 1$ \\
\hline Average & 1398 & $45 \cdot 1$ \\
\hline Large & 646 & $20 \cdot 8$ \\
\hline \multicolumn{3}{|l|}{ Place of delivery ( $n$ 3101) } \\
\hline Home & 1902 & $61 \cdot 3$ \\
\hline Health facility & 1199 & $38 \cdot 7$ \\
\hline \multicolumn{3}{|l|}{ Mode of delivery ( $n$ 3101) } \\
\hline Non-Caesarean & 2823 & $91 \cdot 0$ \\
\hline Caesarean section & 278 & $9 \cdot 0$ \\
\hline Type of delivery assistance ( $n$ 3074) & & \\
\hline Health professional & 1183 & $38 \cdot 5$ \\
\hline Traditional birth attendant & 1046 & $34 \cdot 0$ \\
\hline Other untrained & 845 & $27 \cdot 5$ \\
\hline Antenatal clinic visits ( $n$ 3091) & & \\
\hline None & 1005 & $32 \cdot 5$ \\
\hline $1-3$ & 1162 & $37 \cdot 6$ \\
\hline$\geq 4$ & 924 & $29 \cdot 9$ \\
\hline Timing of postnatal check-up ( $n 117$ & & \\
\hline Day 0-2 & 395 & $33 \cdot 7$ \\
\hline Day 3-6 & 684 & $58 \cdot 4$ \\
\hline Day 7 or later & 72 & $6 \cdot 2$ \\
\hline No check-ups (including missing) & 21 & $1 \cdot 8$ \\
\hline Household level factors & & \\
\hline Household wealth index & & \\
\hline Poorest & 683 & $22 \cdot 0$ \\
\hline Poorer & 670 & $21 \cdot 6$ \\
\hline Middle & 625 & $20 \cdot 2$ \\
\hline
\end{tabular}

Table 1 Continued

\begin{tabular}{lcc}
\hline Characteristic & $n$ & $\%$ \\
\hline Richer & 582 & $18 \cdot 8$ \\
$\quad$ Richest & 543 & $17 \cdot 5$ \\
Community-level factors & & \\
$\quad$ Residence & & \\
$\quad$ Urban & 937 & $30 \cdot 2$ \\
$\quad$ Rural & 2166 & $69 \cdot 8$ \\
Geographical region (province) & 1741 & $56 \cdot 1$ \\
$\quad$ Punjab & 775 & $25 \cdot 0$ \\
$\quad$ Sindh & 452 & $14 \cdot 6$ \\
$\quad$ North West Frontier & 136 & $4 \cdot 4$ \\
$\quad$ Balochistan & \\
\hline
\end{tabular}

Weighted total was 3103 unless stated otherwise within parentheses. *Divorced, separated and widowed.

worked in the 12 months prior to the survey. Nearly $64 \%$ $(63.8 \%)$ of the mothers had no education while just over a third $(34.7 \%)$ of the fathers were uneducated. The majority of the mothers $(77 \cdot 6 \%)$ were aged $20-34$ years. Both male and female children, and different age categories, were nearly equally represented in the sample. One-third of the mothers $(32.5 \%)$ had no ANC visits during the pregnancy. Of the total births, almost two-thirds (61.3\%) were delivered at home and $27.5 \%$ of the deliveries were conducted by untrained personnel. According to the mothers' perception, $45.1 \%$ of the children were of average size at birth while $34 \cdot 1 \%$ were of small size. About $70 \%$ (69.8\%) of the children were living in rural areas. Details can be seen in Table 1.

Regardless of being breast-fed or not, 997 (32.1\% (95\% CI $30 \cdot 0,34 \cdot 4) \%$ infants received food or drink from a bottle with a nipple or teat during the $24 \mathrm{~h}$ prior to the interview. Rate of timely initiation of breast-feeding was $27 \cdot 3 \%$ in children aged $0-23$ months. The bottle-feeding rate varied with age; it was $26 \cdot 8 \%$ in infants aged $<6$ months, $36 \cdot 8 \%$ in infants aged $6-11$ months and $33 \cdot 2 \%$ in children 12-23 months of age. The EBF rate in children aged $<6$ months was $37 \cdot 1 \%(95 \%$ CI $33 \cdot 4,40 \cdot 9) \%$, while the PBF rate in children $<6$ months of age was $18.7 \%$ (95\% CI $15 \cdot 8,22 \cdot 1) \%$ (Table 2). Figure 1 indicates that the PBF rate increased consistently as the EBF rate decreased from infants $<1$ month of age $(12 \cdot 3 \% v .58 \cdot 8 \%)$ to infants at $5-5.9$ months of age $(23 \cdot 8 \% v \cdot 16 \cdot 8 \%)$. Figure 2 shows the distribution of children by breast-feeding status according to child age.

The median duration of EBF was 1.9 months. The rates of related breast-feeding indicators among children aged 0-23 months are shown in Table 2.

\section{Univariate analyses}

Timely initiation of breast-feeding

Working mothers (20.7\%, $P<0 \cdot 001)$, mothers who delivered by Caesarean section $(17 \cdot 0 \%, P=0 \cdot 001)$ and those residing in Sindh Province $(17 \cdot 6 \%, P<0 \cdot 001)$ had significantly lower rates of timely initiation of breast-feeding (Table 3 ). 
Table 2 Breast-feeding indicators among children aged 0-23 months ( $n$ 3103); secondary analysis of data from the Pakistan Demographic and Health Survey (PDHS) 2006-2007

\begin{tabular}{|c|c|c|c|c|}
\hline Indicator & Sample size (weighted) & $n$ (weighted) & Rate (\%) & $95 \% \mathrm{Cl}$ \\
\hline Timely initiation of breast-feeding ( $<24$ months) & 3103 & 848 & $27 \cdot 3$ & $25 \cdot 1,29 \cdot 7$ \\
\hline Ever breast-fed ( $<24$ months) & 3103 & 3029 & $97 \cdot 6$ & $96 \cdot 9,98 \cdot 2$ \\
\hline Ever breast-fed ( $<12$ months) & 1766 & 1727 & $97 \cdot 8$ & $96 \cdot 9,98 \cdot 4$ \\
\hline Ever breast-fed (12-23 months) & 1337 & 1302 & $97 \cdot 4$ & $96 \cdot 2,98 \cdot 3$ \\
\hline Exclusive breast-feeding ( $<6$ months) & 955 & 354 & $37 \cdot 1$ & $33 \cdot 4,40 \cdot 9$ \\
\hline Exclusive breast-feeding (0-1 month) & 282 & 154 & $54 \cdot 6$ & $47 \cdot 6,61 \cdot 5$ \\
\hline Exclusive breast-feeding ( $2-3$ months) & 355 & 127 & $35 \cdot 7$ & $30 \cdot 2,41 \cdot 6$ \\
\hline Exclusive breast-feeding (4-5 months) & 319 & 74 & $23 \cdot 1$ & $18 \cdot 4,28 \cdot 6$ \\
\hline Exclusive breast-feeding (0-3 months) & 637 & 281 & $44 \cdot 1$ & $39 \cdot 5,48 \cdot 8$ \\
\hline Predominant breast-feeding ( $<6$ months) & 955 & 179 & $18 \cdot 7$ & $15 \cdot 8,22 \cdot 1$ \\
\hline Bottle-feeding ( $<24$ months) & 3103 & 997 & $32 \cdot 1$ & $30 \cdot 0,34 \cdot 4$ \\
\hline Bottle-feeding (<6 months) & 955 & 256 & $26 \cdot 8$ & $23 \cdot 5,30 \cdot 3$ \\
\hline Bottle-feeding (6-11 months) & 811 & 298 & $36 \cdot 8$ & $32 \cdot 6,41 \cdot 1$ \\
\hline Bottle-feeding (12-23 months) & 1337 & 444 & $33 \cdot 2$ & $30 \cdot 2,36 \cdot 3$ \\
\hline Median duration of exclusive breast-feeding ( $<36$ months) (months) & \multicolumn{4}{|c|}{$1 \cdot 9$} \\
\hline
\end{tabular}

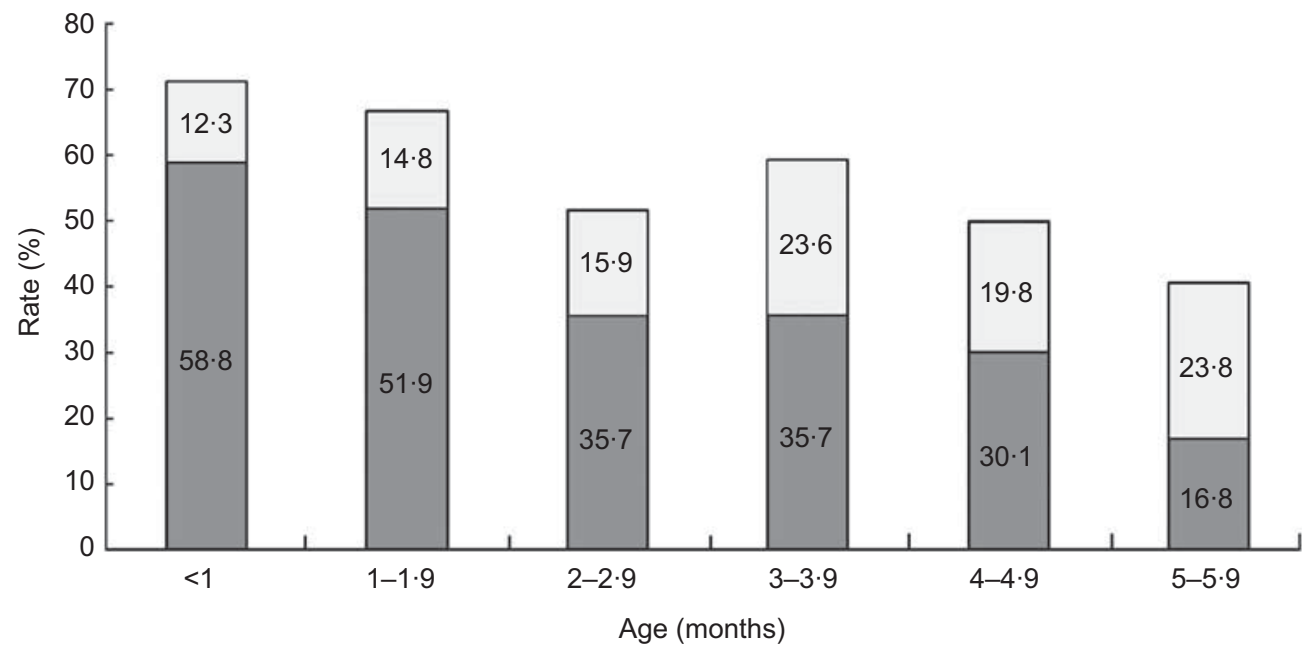

Fig 1 Rates of exclusive breast-feeding $(\square)$ and predominant breast-feeding ( $\square$ ) according to child's age; secondary analysis of data from the Pakistan Demographic and Health Survey (PDHS) 2006-2007

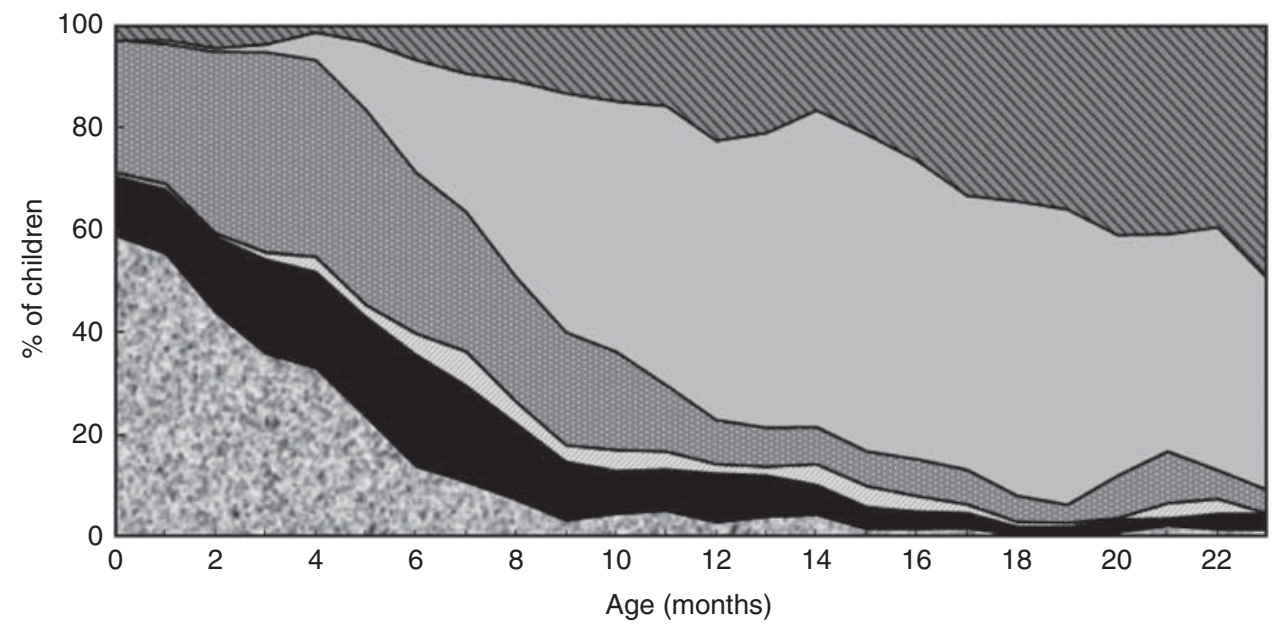

Fig 2 Distribution of children by breast-feeding (BF) status ( $\square$, exclusive BF; $\square, B F+$ water; $\square, B F+$ liquids/juice; 圆, BF+other milk; $\square$, BF+complementary foods; $\mathbb{Q}$, no BF) according to child's age; secondary analysis of data from the Pakistan Demographic and Health Survey (PDHS) 2006-2007 
Table 3 Rates of timely initiation of breast-feeding, bottle-feeding, exclusive breast-feeding and predominant breast-feeding by individual-, household- and community-level characteristics; secondary analysis of data from the Pakistan Demographic and Health Survey (PDHS) 2006-2007

\begin{tabular}{|c|c|c|c|c|c|c|c|c|c|c|c|c|}
\hline \multirow[b]{2}{*}{ Characteristic } & \multicolumn{3}{|c|}{$\begin{array}{l}\text { Timely initiation of breast-feeding } \\
\qquad(<24 \text { months })\end{array}$} & \multicolumn{3}{|c|}{$\begin{array}{l}\text { Bottle-feeding } \\
(<24 \text { months })\end{array}$} & \multicolumn{3}{|c|}{$\begin{array}{l}\text { Exclusive breast-feeding } \\
\qquad(<6 \text { months })\end{array}$} & \multicolumn{3}{|c|}{$\begin{array}{l}\text { Predominant breast-feeding } \\
\text { ( }<6 \text { months) }\end{array}$} \\
\hline & $\%$ & $95 \% \mathrm{Cl}$ & $P$ & $\%$ & $95 \% \mathrm{Cl}$ & $P$ & $\%$ & $95 \% \mathrm{Cl}$ & $P$ & $\%$ & $95 \% \mathrm{Cl}$ & $P$ \\
\hline \multicolumn{13}{|l|}{ Individual-level factors } \\
\hline \multicolumn{13}{|l|}{ Maternal working status } \\
\hline Non-working & $29 \cdot 2$ & $26 \cdot 61,31 \cdot 83$ & \multirow[t]{2}{*}{$<0.001$} & $33 \cdot 6$ & $31 \cdot 30,36 \cdot 03$ & \multirow[t]{2}{*}{$0 \cdot 012$} & $39 \cdot 1$ & $34 \cdot 97,43 \cdot 47$ & \multirow[t]{2}{*}{0.010} & $17 \cdot 4$ & $14 \cdot 36,21 \cdot 02$ & \multirow[t]{2}{*}{0.058} \\
\hline Working (past 12 months) & $20 \cdot 7$ & $17 \cdot 36,24 \cdot 42$ & & $27 \cdot 2$ & $23 \cdot 11,31 \cdot 78$ & & $26 \cdot 3$ & $19 \cdot 63,34 \cdot 32$ & & $25 \cdot 5$ & $18 \cdot 76,33 \cdot 75$ & \\
\hline \multicolumn{13}{|l|}{ Maternal education } \\
\hline No education & $27 \cdot 2$ & $24 \cdot 44,30 \cdot 24$ & \multirow[t]{3}{*}{0.922} & $25 \cdot 3$ & $22 \cdot 84,27 \cdot 82$ & \multirow[t]{3}{*}{$<0.001$} & $37 \cdot 3$ & $32 \cdot 74,42 \cdot 12$ & \multirow[t]{3}{*}{0.950} & $21 \cdot 9$ & $18 \cdot 19,26 \cdot 07$ & \multirow[t]{3}{*}{$0 \cdot 016$} \\
\hline Primary & $26 \cdot 7$ & $21 \cdot 94,32 \cdot 10$ & & $39 \cdot 2$ & $34.05,44.69$ & & $37 \cdot 6$ & $28 \cdot 95,47 \cdot 13$ & & $14 \cdot 4$ & $8 \cdot 93,22 \cdot 49$ & \\
\hline Secondary and above & $28 \cdot 0$ & $24 \cdot 06,32 \cdot 32$ & & $48 \cdot 0$ & $43 \cdot 39,52 \cdot 69$ & & $36 \cdot 0$ & $28 \cdot 29,44 \cdot 57$ & & $11 \cdot 9$ & $7 \cdot 30,18 \cdot 84$ & \\
\hline \multicolumn{13}{|l|}{ Literacy } \\
\hline Cannot read at all & $27 \cdot 0$ & $24 \cdot 16,29 \cdot 94$ & \multirow[t]{2}{*}{0.828} & $25 \cdot 3$ & $22 \cdot 88,27 \cdot 89$ & \multirow[t]{2}{*}{$<0.001$} & $36 \cdot 3$ & $31 \cdot 80,41 \cdot 09$ & $0 \cdot 410$ & $21 \cdot 9$ & $18 \cdot 22,26 \cdot 15$ & 0.010 \\
\hline Able to read only passage & $28 \cdot 0$ & $24 \cdot 80,31 \cdot 34$ & & $43 \cdot 7$ & $40 \cdot 28,47 \cdot 15$ & & $38 \cdot 2$ & $32 \cdot 17,44 \cdot 51$ & & $13 \cdot 2$ & $9 \cdot 31,18 \cdot 44$ & \\
\hline Mother's age (years) & & & & & & & & & & & & \\
\hline $15-19$ & $29 \cdot 8$ & $22 \cdot 61,38 \cdot 18$ & 0.802 & $29 \cdot 1$ & $21 \cdot 72,37 \cdot 72$ & 0.754 & $34 \cdot 5$ & $24 \cdot 34,46 \cdot 34$ & 0.900 & $22 \cdot 8$ & $13 \cdot 97,35 \cdot 07$ & 0.552 \\
\hline $20-34$ & $27 \cdot 3$ & $24 \cdot 79,29 \cdot 87$ & & $32 \cdot 3$ & $29 \cdot 97,34 \cdot 73$ & & $37 \cdot 2$ & $33 \cdot 10,41 \cdot 57$ & & $18 \cdot 8$ & $15 \cdot 59,22 \cdot 45$ & \\
\hline $35-49$ & $26 \cdot 8$ & $22 \cdot 25,31 \cdot 89$ & & $32 \cdot 4$ & $27 \cdot 70,37 \cdot 48$ & & $37 \cdot 7$ & $28 \cdot 75,47 \cdot 63$ & & $16 \cdot 3$ & $10 \cdot 53,24 \cdot 23$ & \\
\hline Marital status & & & & & & & & & & & & \\
\hline Currently married & $27 \cdot 3$ & $25 \cdot 09,29 \cdot 68$ & 0.952 & $32 \cdot 1$ & $30 \cdot 00,34 \cdot 35$ & 0.929 & $37 \cdot 1$ & $33 \cdot 45,40 \cdot 93$ & 0.440 & $18 \cdot 7$ & $15 \cdot 78,22 \cdot 01$ & 0.037 \\
\hline Formerly married* & $26 \cdot 6$ & $10 \cdot 23,53 \cdot 61$ & & $33 \cdot 3$ & $13 \cdot 83,60 \cdot 77$ & & $0 \cdot 0$ & & & $0 \cdot 0$ & & \\
\hline Birth order & & & & & & & & & & & & \\
\hline First-born & $24 \cdot 9$ & $21 \cdot 00,29 \cdot 27$ & $0 \cdot 189$ & $35 \cdot 1$ & $30 \cdot 58,39 \cdot 92$ & 0.347 & $34 \cdot 4$ & $27 \cdot 17,42 \cdot 49$ & 0.050 & $23 \cdot 2$ & $16 \cdot 97,30 \cdot 84$ & 0.077 \\
\hline Second- to fourth-born & $26 \cdot 9$ & $24 \cdot 04,29 \cdot 85$ & & $31 \cdot 7$ & $28 \cdot 71,34 \cdot 86$ & & $34 \cdot 3$ & $29 \cdot 45,39 \cdot 54$ & & $19 \cdot 6$ & $15 \cdot 57,24 \cdot 28$ & \\
\hline Fifth-born or higher & $29 \cdot 6$ & $26 \cdot 00,33 \cdot 43$ & & $31 \cdot 0$ & $27 \cdot 51,34 \cdot 65$ & & $44 \cdot 3$ & $37 \cdot 16,51 \cdot 64$ & & $13 \cdot 7$ & $9 \cdot 47,19 \cdot 50$ & \\
\hline Preceding birth interval (mont & & & & & & & & & & & & \\
\hline No previous birth & $24 \cdot 9$ & $21 \cdot 00,29 \cdot 27$ & 0.488 & $35 \cdot 1$ & $30 \cdot 58,39 \cdot 92$ & $0 \cdot 169$ & $34 \cdot 4$ & $27 \cdot 17,42 \cdot 49$ & 0.020 & $23 \cdot 2$ & $16 \cdot 97,30 \cdot 84$ & 0.244 \\
\hline $0-14$ months & $29 \cdot 7$ & $22 \cdot 14,38 \cdot 55$ & & $35 \cdot 8$ & $28 \cdot 50,43 \cdot 82$ & & $22 \cdot 8$ & $12 \cdot 82,37 \cdot 18$ & & $20 \cdot \overline{8}$ & $11 \cdot 22,35 \cdot 22$ & \\
\hline 14-24 months & $27 \cdot 0$ & $23 \cdot 14,31 \cdot 27$ & & $33 \cdot 1$ & $28 \cdot 85,37 \cdot 65$ & & $31 \cdot 2$ & $23 \cdot 62,39 \cdot 86$ & & $21 \cdot 2$ & $15 \cdot 10,29 \cdot 01$ & \\
\hline$\geq 25$ months & $28 \cdot 2$ & $25 \cdot 31,31 \cdot 33$ & & $30 \cdot 1$ & $27 \cdot 33,32 \cdot 98$ & & $42 \cdot 1$ & $36.94,47.44$ & & $18 \cdot 8$ & $12 \cdot 28,20 \cdot 08$ & \\
\hline Sex of baby & & & & & & & & & & & & \\
\hline Male & $26 \cdot 7$ & $23 \cdot 99,29 \cdot 66$ & 0.497 & $32 \cdot 8$ & $30 \cdot 03,35 \cdot 73$ & 0.471 & $38 \cdot 9$ & $34 \cdot 15,43 \cdot 97$ & $0 \cdot 270$ & $18 \cdot 4$ & $14 \cdot 53,23 \cdot 06$ & 0.829 \\
\hline Female & $28 \cdot 0$ & $25 \cdot 06,31 \cdot 10$ & & $31 \cdot 4$ & $28 \cdot 50,34 \cdot 42$ & & $35 \cdot 0$ & $29 \cdot 91,40 \cdot 54$ & & $19 \cdot 1$ & $15 \cdot 01,23 \cdot 95$ & \\
\hline Age of child (months) & & & & & & & & & & & & \\
\hline $0-5$ & $25 \cdot 7$ & $22 \cdot 25,29 \cdot 57$ & $0 \cdot 341$ & $26 \cdot 8$ & $23 \cdot 48,30 \cdot 33$ & 0.001 & & & & $18 \cdot 7$ & $14 \cdot 80,22 \cdot 15$ & \\
\hline $11-6$ & $26 \cdot 5$ & $22 \cdot 93,30 \cdot 46$ & & $36 \cdot 8$ & $32 \cdot 57,41 \cdot 14$ & & & & & & & \\
\hline $12-17$ & $30 \cdot 0$ & $26 \cdot 38,33 \cdot 97$ & & $34 \cdot 3$ & $30 \cdot 56,38 \cdot 28$ & & & & & & & \\
\hline $18-23$ & $27 \cdot 1$ & $22 \cdot 51,32 \cdot 12$ & & $31 \cdot 3$ & $26 \cdot 82,36 \cdot 06$ & & & & & & & \\
\hline Perceived birth size of baby & & & & & & & & & & & & \\
\hline Small & $25 \cdot 9$ & $22 \cdot 57,29 \cdot 44$ & $0 \cdot 150$ & $31 \cdot 2$ & $27 \cdot 86,34 \cdot 69$ & $0 \cdot 771$ & $36 \cdot 7$ & $31 \cdot 04,42 \cdot 73$ & $0 \cdot 800$ & $15 \cdot 6$ & $11 \cdot 40,20 \cdot 88$ & 0.272 \\
\hline Average & $26 \cdot 7$ & $23 \cdot 67,29 \cdot 87$ & & $32 \cdot \overline{6}$ & $29 \cdot 64,35 \cdot 79$ & & $38 \cdot 0$ & $32 \cdot 27,44 \cdot 11$ & & $20 \cdot 7$ & $16 \cdot 25,25 \cdot 90$ & \\
\hline Large & $31 \cdot 0$ & $25 \cdot 95,36 \cdot 55$ & & $32 \cdot 6$ & $27 \cdot 97,37 \cdot 49$ & & $36 \cdot 1$ & $28 \cdot 09,44 \cdot 93$ & & $20 \cdot 8$ & $14 \cdot 98,28 \cdot 13$ & \\
\hline Place of delivery & & & & & & & & & & & & \\
\hline Home & $28 \cdot 1$ & $25 \cdot 30,31 \cdot 01$ & 0.464 & $26 \cdot 2$ & $23 \cdot 70,28 \cdot 91$ & $<0.001$ & $37 \cdot 6$ & $32 \cdot 90,42 \cdot 52$ & 0.630 & $22 \cdot 7$ & $18 \cdot 70,27 \cdot 37$ & 0.002 \\
\hline Health facility & $26 \cdot 2$ & $23 \cdot 09,29 \cdot 52$ & & $41 \cdot 6$ & $38 \cdot 11,45 \cdot 14$ & & $36 \cdot 5$ & $30 \cdot 76,42 \cdot 67$ & & $12 \cdot 5$ & $9 \cdot 17,16 \cdot 89$ & \\
\hline Mode of delivery & & & & & & & & & & & & \\
\hline Non-Caesarean & $28 \cdot 4$ & $25 \cdot 97,30 \cdot 85$ & 0.001 & $30 \cdot 6$ & $28 \cdot 44,32 \cdot 87$ & $<0.001$ & $37 \cdot 3$ & $33 \cdot 54,41 \cdot 23$ & $0 \cdot 730$ & $19 \cdot 8$ & $16 \cdot 69,23 \cdot 36$ & 0.002 \\
\hline Caesarean section & $17 \cdot 0$ & $12 \cdot 34,22 \cdot 99$ & & $47 \cdot 9$ & $40 \cdot 85,55 \cdot 04$ & & $35 \cdot 2$ & $23 \cdot 26,49 \cdot 29$ & & $5 \cdot 4$ & $1 \cdot 92,14 \cdot 18$ & \\
\hline
\end{tabular}




\begin{tabular}{|c|c|c|c|c|c|c|c|c|c|c|c|c|}
\hline \multirow[b]{2}{*}{ Characteristic } & \multicolumn{3}{|c|}{$\begin{array}{l}\text { Timely initiation of breast-feeding } \\
\qquad(<24 \text { months })\end{array}$} & \multicolumn{3}{|c|}{$\begin{array}{l}\text { Bottle-feeding } \\
\text { (<24 months) }\end{array}$} & \multicolumn{3}{|c|}{$\begin{array}{l}\text { Exclusive breast-feeding } \\
\qquad(<6 \text { months })\end{array}$} & \multicolumn{3}{|c|}{$\begin{array}{l}\text { Predominant breast-feeding } \\
\text { ( }<6 \text { months })\end{array}$} \\
\hline & $\%$ & $95 \% \mathrm{Cl}$ & $P$ & $\%$ & $95 \% \mathrm{Cl}$ & $P$ & $\%$ & $95 \% \mathrm{Cl}$ & $P$ & $\%$ & $95 \% \mathrm{Cl}$ & $P$ \\
\hline \multicolumn{13}{|l|}{ Type of delivery assistance } \\
\hline Health professional & $26 \cdot 5$ & $23 \cdot 52,29 \cdot 76$ & \multirow[t]{3}{*}{$0 \cdot 745$} & $40 \cdot 4$ & $36 \cdot 77,44 \cdot 04$ & \multirow[t]{3}{*}{$<0.001$} & $37 \cdot 6$ & $31 \cdot 70,43 \cdot 93$ & \multirow[t]{3}{*}{0.330} & $12 \cdot 7$ & $9 \cdot 27,17 \cdot 19$ & \multirow[t]{3}{*}{0.002} \\
\hline Traditional birth attendant & $28 \cdot 1$ & $24 \cdot 61,31.97$ & & $30 \cdot 5$ & $27 \cdot 17,33.97$ & & $33 \cdot 0$ & $27 \cdot 48,38 \cdot 97$ & & $25 \cdot 5$ & $20 \cdot 27,31 \cdot 45$ & \\
\hline Other & $27 \cdot 7$ & $23 \cdot 92,31 \cdot 81$ & & $23 \cdot 1$ & $19 \cdot 76,26 \cdot 69$ & & $41 \cdot 4$ & $34 \cdot 27,48 \cdot 83$ & & $19 \cdot 2$ & $14 \cdot 00,25 \cdot 83$ & \\
\hline \multicolumn{13}{|l|}{ Antenatal clinic visits } \\
\hline None & $28 \cdot 4$ & $24 \cdot 86,32 \cdot 31$ & \multirow[t]{3}{*}{0.604} & $21 \cdot 9$ & $18 \cdot 95,25 \cdot 24$ & \multirow[t]{3}{*}{$<0.001$} & $40 \cdot 4$ & $34 \cdot 34,46 \cdot 81$ & \multirow[t]{3}{*}{$0 \cdot 270$} & $21 \cdot 8$ & $16 \cdot 65,27 \cdot 89$ & \multirow[t]{3}{*}{$0 \cdot 249$} \\
\hline $1-3$ & $27 \cdot 4$ & $24 \cdot 15,30 \cdot 81$ & & $29 \cdot 4$ & $26 \cdot 39,32 \cdot 65$ & & $37 \cdot 3$ & $31 \cdot 81,43 \cdot 06$ & & $19 \cdot 0$ & $14 \cdot 56,24 \cdot 29$ & \\
\hline$\geq 4$ & $25 \cdot 9$ & $22 \cdot 59,29 \cdot 60$ & & $46 \cdot 2$ & $42 \cdot 39,50 \cdot 12$ & & $33 \cdot 4$ & $27 \cdot 02,40 \cdot 37$ & & $15 \cdot 2$ & $10 \cdot 85,20 \cdot 84$ & \\
\hline \multicolumn{13}{|l|}{ Timing of postnatal check-up } \\
\hline Day 0-2 & $26 \cdot 1$ & $21 \cdot 12,31 \cdot 66$ & \multirow[t]{4}{*}{$0 \cdot 860$} & $37 \cdot 2$ & $31 \cdot 11,43 \cdot 67$ & \multirow[t]{4}{*}{$<0.001$} & $41 \cdot 8$ & $31 \cdot 50,52 \cdot 80$ & \multirow[t]{4}{*}{$0 \cdot 790$} & $13 \cdot 0$ & $7 \cdot 59,21 \cdot 30$ & \multirow[t]{4}{*}{0.023} \\
\hline Day 3-6 & $27 \cdot 5$ & $23 \cdot 55,31 \cdot 84$ & & $43 \cdot 8$ & $39 \cdot 49,48 \cdot 20$ & & $33 \cdot 8$ & $26 \cdot 77,41 \cdot 70$ & & $11 \cdot 7$ & $7 \cdot 63,17 \cdot 60$ & \\
\hline Day 7 or later & $21 \cdot 8$ & $12 \cdot 61,35 \cdot 09$ & & $35 \cdot 1$ & $23 \cdot 82,48 \cdot 34$ & & $35 \cdot 3$ & $18 \cdot 12,57 \cdot 27$ & & $13 \cdot 6$ & $4.92,32 \cdot 53$ & \\
\hline Missing/no check-ups & $22 \cdot 2$ & $7 \cdot 195,51 \cdot 28$ & & $59 \cdot 0$ & $33 \cdot 83,80 \cdot 22$ & & $30 \cdot 4$ & $6 \cdot 75,72 \cdot 50$ & & $32 \cdot 2$ & $5 \cdot 23,80 \cdot 28$ & \\
\hline \multicolumn{13}{|l|}{ Household-level factors } \\
\hline \multicolumn{13}{|l|}{ Household wealth index } \\
\hline Poorest & $24 \cdot 1$ & $19 \cdot 80,29 \cdot 05$ & \multirow[t]{5}{*}{0.421} & $18 \cdot 1$ & $14 \cdot 59,22 \cdot 25$ & \multirow[t]{5}{*}{$<0.001$} & $37 \cdot 9$ & $30 \cdot 38,46 \cdot 07$ & \multirow[t]{5}{*}{$0 \cdot 130$} & $27 \cdot 6$ & $21 \cdot 04,35 \cdot 24$ & \multirow[t]{5}{*}{0.002} \\
\hline Poorer & $26 \cdot 9$ & $22 \cdot 42,31 \cdot 98$ & & $27 \cdot 4$ & $23 \cdot 60,31 \cdot 65$ & & $43 \cdot 3$ & $35 \cdot 80,51 \cdot 02$ & & $21 \cdot 6$ & $15 \cdot 94,28 \cdot 48$ & \\
\hline Middle & $28 \cdot 0$ & $23 \cdot 77,32 \cdot 70$ & & $31 \cdot 1$ & $26 \cdot 86,35 \cdot 76$ & & $39 \cdot 3$ & $31 \cdot 16,48 \cdot 09$ & & $11 \cdot 2$ & $6 \cdot 82,17 \cdot 70$ & \\
\hline Richer & $30 \cdot 7$ & $25 \cdot 96,35 \cdot 86$ & & $38 \cdot 4$ & $33 \cdot 85,43 \cdot 20$ & & $34 \cdot 4$ & $26 \cdot 70,43 \cdot 09$ & & $18 \cdot 4$ & $12 \cdot 36,26 \cdot 53$ & \\
\hline Richest & $27 \cdot 4$ & $22 \cdot 49,32 \cdot 92$ & & $50 \cdot 0$ & $45 \cdot 08,55 \cdot 00$ & & $28 \cdot 5$ & $21 \cdot 27,36 \cdot 92$ & & $12 \cdot 6$ & $7 \cdot 774,19 \cdot 88$ & \\
\hline \multirow{2}{*}{\multicolumn{13}{|c|}{ Community-level factors }} \\
\hline & & & & & & & & & & & & \\
\hline Urban & $26 \cdot 9$ & $23 \cdot 36,30 \cdot 78$ & $0 \cdot 807$ & $40 \cdot 5$ & $36 \cdot 61,44 \cdot 55$ & $<0.001$ & $35 \cdot 7$ & $29 \cdot 13,42 \cdot 91$ & 0.640 & $13 \cdot 4$ & $9 \cdot 31,18 \cdot 89$ & 0.022 \\
\hline Rural & $27 \cdot 5$ & $24 \cdot 70,30 \cdot 49$ & & $28 \cdot 5$ & $26 \cdot 07,31 \cdot 10$ & & $37 \cdot 7$ & $33 \cdot 36,42 \cdot 27$ & & $21 \cdot 1$ & $17 \cdot 47,25 \cdot 31$ & \\
\hline Geographical region (provinc & & & & & & & & & & & & \\
\hline Punjab & $29 \cdot 0$ & $25 \cdot 77,32 \cdot 38$ & $<0.001$ & $38 \cdot 3$ & $35 \cdot 07,41 \cdot 59$ & $<0.001$ & $32 \cdot 5$ & $27 \cdot 55,37 \cdot 88$ & 0.001 & $19 \cdot 7$ & $15 \cdot 46,24 \cdot 79$ & 0.827 \\
\hline Sindh & $17 \cdot 6$ & $14 \cdot 43,21 \cdot 37$ & & $26 \cdot 3$ & $22 \cdot 79,30 \cdot 11$ & & $37 \cdot 2$ & $30 \cdot 59,44 \cdot 23$ & & $17 \cdot 5$ & $13 \cdot 09,22 \cdot 92$ & \\
\hline North West Frontier & $34 \cdot 2$ & $28 \cdot 36,40 \cdot 47$ & & $22 \cdot 5$ & $18 \cdot 13,27 \cdot 57$ & & $55 \cdot 2$ & $46 \cdot 39,63 \cdot 72$ & & $18 \cdot 7$ & $12 \cdot 51,26 \cdot 95$ & \\
\hline Balochistan & $38 \cdot 8$ & $28 \cdot 21,50 \cdot 47$ & & $19 \cdot 0$ & $14 \cdot 36,24 \cdot 61$ & & $35 \cdot 6$ & $24 \cdot 73,48 \cdot 14$ & & $14 \cdot 1$ & $5 \cdot 42,32 \cdot 05$ & \\
\hline
\end{tabular}

*Divorced, separated and widowed. 


\section{Exclusive breast-feeding}

EBF rates were significantly lower among working mothers (26.3\%, $P=0 \cdot 010)$, mothers with a preceding birth interval of $<15$ months $(22 \cdot 8 \%, P=0 \cdot 020)$ and mothers living in Punjab Province $(32 \cdot 5 \%, P=0 \cdot 001$; Table 3$)$.

\section{Bottle-feeding}

The bottle-feeding rates were significantly higher among non-working women $(33.6 \%, P=0.012)$ and those who were literate $(43.7 \%, P<0 \cdot 001)$, especially with secondary and higher education level (48.0\%, $P<0 \cdot 001)$. Women who had four or more ANC visits $(46 \cdot 2 \%, P<0 \cdot 001)$, delivered at a health facility $(41.6 \%, P<0 \cdot 001)$, delivered by a health professional $(40 \cdot 4 \%, P<0 \cdot 001)$, who underwent a Caesarean section $(47.9 \%, P<0.001)$ and who had no PNC (59.0\%, $P<0.001)$ had significantly higher bottle-feeding rates. Infants aged 6-11 months (36.8\%, $P=0 \cdot 001)$, in the richest household wealth index quintile (50.0\%, $P<0 \cdot 001)$, of urban residence $(40.5 \%, P<0 \cdot 001)$ and residing in Punjab Province $(38.3 \%, P<0 \cdot 001)$ also had significantly higher bottle-feeding rates (Table 3 ).

\section{Predominant breast-feeding}

PBF rates were significantly higher among mothers who had no education $(21.9 \%, P=0.016)$ compared with those who had primary, secondary or higher education. PBF rates were also higher among women whose deliveries were assisted by traditional birth attendants (25.5\%, $P=0.002)$. Mothers from rural regions $(21 \cdot 1 \%$, $P=0.022)$ and those from the poorest households (27.6\%, $P=0.002)$ reported significantly higher PBF rates (Table 3 ).

\section{Multivariate analyses}

No timely initiation of breast-feeding

Working mothers $(\mathrm{AOR}=1 \cdot 48,95 \%$ CI $1 \cdot 16,1 \cdot 87$; $P=0 \cdot 001)$, mothers who delivered by Caesarean section (AOR $=1 \cdot 95,95 \%$ CI $1 \cdot 30,2 \cdot 90 ; P=0 \cdot 001)$ and those residing in Sindh Province $(\mathrm{AOR}=1 \cdot 88,95 \%$ CI $1 \cdot 40$, $2 \cdot 51 ; P<0 \cdot 001)$ had higher odds for no timely initiation of breast-feeding (Table 4).

\section{Bottle-feeding}

The odds of an infant being bottle-fed was higher when the father's education was secondary or above (AOR $=1 \cdot 41$, 95\% CI $1.05,1 \cdot 89 ; P=0.023)$, with increasing age of the child $($ AOR $=1 \cdot 02,95 \%$ CI $1 \cdot 01,1 \cdot 03 ; P=0 \cdot 006)$ and for women who had four or more ANC visits $(\mathrm{AOR}=1.93$, 95\% CI $1.46,2 \cdot 55 ; P<0 \cdot 001)$. Compared with mothers from the poorest households, those belonging to the richest households (AOR $=2 \cdot 41,95 \%$ CI 1.62, 3.58; $P<0 \cdot 001$ ) had higher odds of bottle-feeding their babies. Compared with Punjab Province, mothers who lived in other provinces were significantly less likely to bottle-feed their babies (all $P<0 \cdot 001$; Table 4).

\section{Exclusive breast-feeding}

Non-EBF was significantly more likely for working mothers $(\mathrm{AOR}=1 \cdot 76,95 \%$ CI $1 \cdot 13,2 \cdot 75 ; P=0 \cdot 013)$, infants whose mothers belonged to the highest household wealth index quintile (AOR $=2 \cdot 31,95 \%$ CI $1 \cdot 22$, $4 \cdot 36 ; P=0 \cdot 010)$ and for mothers who lived in Punjab Province. As expected, increasing age of the infant was associated with significantly more non-EBF (AOR $=1 \cdot 46$, $95 \%$ CI $1 \cdot 32,1 \cdot 62 ; P<0 \cdot 001)$. Mothers who were able to read a passage of text were less likely not to breast-feed their babies exclusively (AOR $=0 \cdot 64,95 \%$ CI 0.43, 0.96; $P=0.029)$ than mothers who were not able to read at all (Table 5).

\section{Predominant breast-feeding}

The practice of PBF was significantly less common in mothers who were literate $(\mathrm{AOR}=0 \cdot 58,95 \% \mathrm{CI} 0 \cdot 35$, 0.95; $P=0.033)$. PBF had a higher odds ratio when the delivery was assisted by a traditional birth attendant (AOR $=1 \cdot 96,95 \%$ CI $1 \cdot 18,3 \cdot 24 ; P=0 \cdot 009)$ as compared with a qualified health professional (Table 5 ).

When compared with bottle-feeding (only children $<6$ months of age), working mothers were more significantly likely to predominantly breast-feed their babies $(\mathrm{AOR}=$ $2 \cdot 26,95 \%$ CI $1 \cdot 22,4 \cdot 18 ; P=0 \cdot 009)$. On the other hand, significantly lower rates of $\mathrm{PBF}$ were reported in mothers who were delivered at a health facility $(\mathrm{AOR}=0 \cdot 47,95 \% \mathrm{CI}$ $0 \cdot 27,0 \cdot 82 ; P=0 \cdot 009)$ and who delivered by Caesarean section $(\mathrm{AOR}=0 \cdot 19,95 \% \mathrm{CI} 0 \cdot 06,0 \cdot 65 ; P=0 \cdot 008)$.

\section{Discussion}

Pakistan has the lowest EBF rate, highest bottle-feeding rate but almost similar PBF compared with other countries in South Asia that have similar socio-economic conditions, such as Sri Lanka ${ }^{(17)}$, Bangladesh ${ }^{(18)}$, India ${ }^{(19)}$ and Nepal ${ }^{(20)}$. These rates of EBF, PBF and bottle-feeding are unacceptable and need improvement if Pakistan is to achieve the goals set out by WHO for optimal infant feeding practices. The present secondary data analysis has identified a number of associations with poor feeding practices which have programmatic implications for child health programmes in Pakistan; the more important ones are examined in the discussion below.

The rates of bottle-feeding were significantly higher among women who belonged to higher socio-economic strata with higher levels of education, reflecting the fact that educational status does not necessarily mean greater awareness of the importance of EBF. This trend could have a trickledown effect as these women potentially are role models to which less privileged women aspire. Also of concern was that those women who had four or more ANC visits, who delivered by health-care professionals and who delivered at health-care facilities had much higher bottle-feeding rates. This shows that women who 
Table 4 Survey logistic modelling for no timely initiation of breast-feeding and bottle-feeding (unadjusted and adjusted odds ratios); secondary analysis of data from the Pakistan Demographic and Health Survey (PDHS) 2006-2007

\begin{tabular}{|c|c|c|c|c|c|c|c|c|c|c|c|c|}
\hline \multirow[b]{3}{*}{ Characteristic } & \multicolumn{6}{|c|}{$\begin{array}{l}\text { No timely initiation of breast-feeding } \\
\qquad(<24 \text { months })\end{array}$} & \multicolumn{6}{|c|}{$\begin{array}{l}\text { Bottle-feeding } \\
(<24 \text { months })\end{array}$} \\
\hline & \multicolumn{3}{|c|}{ Unadjusted } & \multicolumn{3}{|c|}{ Adjusted } & \multicolumn{3}{|c|}{ Unadjusted } & \multicolumn{3}{|c|}{ Adjusted } \\
\hline & OR & $95 \% \mathrm{Cl}$ & $P$ & OR & $95 \% \mathrm{Cl}$ & $P$ & OR & $95 \% \mathrm{Cl}$ & $P$ & OR & $95 \% \mathrm{Cl}$ & $P$ \\
\hline \multicolumn{13}{|l|}{ Individual-level factors } \\
\hline \multicolumn{13}{|l|}{ Maternal working status } \\
\hline Non-working & 1.00 & & & 1.00 & & & $1 \cdot 00$ & & & & & \\
\hline Working (past 12 months) & 1.58 & $1 \cdot 25,1.99$ & $<0.001$ & $1 \cdot 48$ & $1 \cdot 16,1 \cdot 87$ & 0.001 & 0.74 & $0.58,0.93$ & 0.011 & & & \\
\hline \multicolumn{13}{|l|}{ Marital status } \\
\hline Currently married & 1.00 & & & & & & $1 \cdot 00$ & & & & & \\
\hline Formerly married* & 1.04 & $0.33,3 \cdot 27$ & 0.952 & & & & $1 \cdot 05$ & $0.34,3 \cdot 28$ & 0.929 & & & \\
\hline \multicolumn{13}{|l|}{ Maternal education } \\
\hline No education & 1.00 & & & & & & 1.00 & & & & & \\
\hline Primary & 1.03 & $0 \cdot 77,1 \cdot 37$ & 0.856 & & & & $1 \cdot 91$ & $1 \cdot 48,2 \cdot 47$ & $<0.001$ & & & \\
\hline Secondary or above & 0.96 & $0.75,1.23$ & 0.759 & & & & $2 \cdot 74$ & $2 \cdot 18,3 \cdot 44$ & $<0.001$ & & & \\
\hline \multicolumn{13}{|l|}{ Literacy } \\
\hline Cannot read at all & 1.00 & & & & & & $1 \cdot 00$ & & & & & \\
\hline Able to read only passage & 0.95 & $0 \cdot 77,1 \cdot 17$ & 0.631 & & & & $2 \cdot 29$ & $1 \cdot 90,2 \cdot 77$ & $<0.001$ & & & \\
\hline \multicolumn{13}{|l|}{ Partner's education } \\
\hline No education & 1.00 & & & & & & 1.00 & & & $1 \cdot 00$ & & \\
\hline Primary & 0.86 & $0 \cdot 65,1 \cdot 13$ & 0.266 & & & & $1 \cdot 35$ & $1 \cdot 02,1 \cdot 77$ & 0.033 & $1 \cdot 31$ & $1 \cdot 00,1 \cdot 74$ & 0.054 \\
\hline Secondary or above & 0.85 & $0.69,1.04$ & $0 \cdot 120$ & & & & $1 \cdot 71$ & $1 \cdot 38,2 \cdot 11$ & $<0.001$ & $1 \cdot 41$ & $1 \cdot 05,1 \cdot 89$ & 0.023 \\
\hline \multicolumn{13}{|l|}{ Mother's age (years) } \\
\hline 15-19 & $1 \cdot 00$ & & & & & & $1 \cdot 00$ & & & & & \\
\hline $20-34$ & $1 \cdot 13$ & $0 \cdot 77,1.67$ & 0.455 & & & & $1 \cdot 16$ & $0.78,1.73$ & 0.455 & & & \\
\hline $35-49$ & $1 \cdot 16$ & $0 \cdot 74,1 \cdot 81$ & 0.514 & & & & $1 \cdot 17$ & $0 \cdot 75,1 \cdot 82$ & 0.490 & & & \\
\hline \multicolumn{13}{|l|}{ Birth order } \\
\hline First-born & $1 \cdot 00$ & & & & & & $1 \cdot 00$ & & & & & \\
\hline Second- to fourth-born & 0.90 & $0 \cdot 71,1 \cdot 16$ & 0.423 & & & & $0 \cdot 86$ & $0 \cdot 67,1 \cdot 11$ & 0.238 & & & \\
\hline Fifth-born or higher & 0.79 & $0.61,1.03$ & 0.077 & & & & $0 \cdot 83$ & $0.64,1.07$ & $0 \cdot 152$ & & & \\
\hline \multicolumn{13}{|l|}{ Preceding birth interval } \\
\hline No previous birth & 1.00 & & & & & & 1.00 & & & & & \\
\hline $0-14$ months & 0.79 & $0.50,1.23$ & 0.293 & & & & $1 \cdot 03$ & $0.69,1.53$ & 0.882 & & & \\
\hline $14-24$ months & 0.90 & $0 \cdot 67,1 \cdot 20$ & 0.461 & & & & 0.91 & $0.69,1.22$ & 0.538 & & & \\
\hline$\geq 25$ months & 0.84 & $0.66,1.07$ & 0.163 & & & & 0.80 & $0.62,1.02$ & 0.067 & & & \\
\hline \multicolumn{13}{|l|}{ Sex of baby } \\
\hline Male & $1 \cdot 00$ & & & & & & $1 \cdot 00$ & & & & & \\
\hline Female & 0.94 & $0 \cdot 78,1 \cdot 13$ & 0.497 & & & & 0.94 & $0 \cdot 78,1 \cdot 12$ & 0.471 & & & \\
\hline Age of child (months) & 0.99 & $0.98,1.00$ & $0 \cdot 159$ & & & & $1 \cdot 02$ & $1.01,1.03$ & 0.005 & $1 \cdot 02$ & $1.01,1.03$ & 0.006 \\
\hline \multicolumn{13}{|l|}{ Perceived birth size of baby } \\
\hline Average & 1.00 & & & & & & 1.00 & & & & & \\
\hline Large & $0 \cdot 81$ & $0 \cdot 61,1 \cdot 07$ & $0 \cdot 143$ & & & & $1 \cdot 00$ & $0 \cdot 78,1 \cdot 28$ & 0.975 & & & \\
\hline Small & 1.04 & $0.83,1 \cdot 31$ & 0.722 & & & & 0.93 & $0 \cdot 76,1 \cdot 15$ & 0.518 & & & \\
\hline \multicolumn{13}{|l|}{ Place of delivery } \\
\hline Home & 1.00 & & & & & & $1 \cdot 00$ & & & & & \\
\hline Health facility & $1 \cdot 10$ & $0.90,1.34$ & 0.350 & & & & $2 \cdot 00$ & $1 \cdot 65,2 \cdot 44$ & $<0.001$ & & & \\
\hline
\end{tabular}


Table 4 Continued

\begin{tabular}{|c|c|c|c|c|c|c|c|c|c|c|c|c|}
\hline \multirow[b]{3}{*}{ Characteristic } & \multicolumn{6}{|c|}{$\begin{array}{l}\text { No timely initiation of breast-feeding } \\
\qquad(<24 \text { months) }\end{array}$} & \multicolumn{6}{|c|}{$\begin{array}{l}\text { Bottle-feeding } \\
(<24 \text { months })\end{array}$} \\
\hline & \multicolumn{3}{|c|}{ Unadjusted } & \multicolumn{3}{|c|}{ Adjusted } & \multicolumn{3}{|c|}{ Unadjusted } & \multicolumn{3}{|c|}{ Adjusted } \\
\hline & OR & $95 \% \mathrm{Cl}$ & $P$ & OR & $95 \% \mathrm{Cl}$ & $P$ & OR & $95 \% \mathrm{Cl}$ & $P$ & OR & $95 \% \mathrm{Cl}$ & $P$ \\
\hline \multicolumn{13}{|l|}{ Mode of delivery } \\
\hline Non-Caesarean & $1 \cdot 00$ & & & $1 \cdot 00$ & & & $1 \cdot 00$ & & & & & \\
\hline Caesarean section & 1.93 & $1 \cdot 31,2 \cdot 84$ & 0.001 & 1.95 & $1 \cdot 30,2 \cdot 90$ & $0 \cdot 001$ & $2 \cdot 08$ & $1 \cdot 54,2 \cdot 81$ & $<0.001$ & & & \\
\hline \multicolumn{13}{|l|}{ Type of delivery assistance } \\
\hline Health professional & $1 \cdot 00$ & & & & & & $1 \cdot 00$ & & & 1.00 & & \\
\hline Traditional birth attendant & 0.92 & $0 \cdot 73,1 \cdot 16$ & 0.486 & & & & 0.65 & $0.52,0.81$ & $<0.001$ & 1.02 & $0 \cdot 80,1 \cdot 31$ & 0.863 \\
\hline Other & 0.94 & $0 \cdot 74,1 \cdot 20$ & 0.629 & & & & 0.44 & $0.35,0.56$ & $<0.001$ & 0.76 & $0.58,0.99$ & 0.039 \\
\hline \multicolumn{13}{|l|}{ Antenatal clinic visits } \\
\hline None & 1.00 & & & & & & $1 \cdot 00$ & & & 1.00 & & \\
\hline $1-3$ & 1.06 & $0.85,1 \cdot 32$ & 0.632 & & & & $1 \cdot 48$ & $1 \cdot 19,1 \cdot 85$ & $<0.001$ & $1 \cdot 21$ & $0.95,1.53$ & $0 \cdot 115$ \\
\hline$\geq 4$ & $1 \cdot 13$ & $0 \cdot 89,1 \cdot 45$ & $0 \cdot 308$ & & & & 3.06 & $2 \cdot 40,3 \cdot 90$ & $<0.001$ & 1.93 & $1 \cdot 46,2 \cdot 55$ & $<0.001$ \\
\hline \multicolumn{13}{|l|}{ Timing of postnatal check-up } \\
\hline Immediate (hospital birth) & $1 \cdot 00$ & & & & & & $1 \cdot 00$ & & & & & \\
\hline Day 0-2 & 0.93 & $0 \cdot 67,1 \cdot 28$ & 0.654 & & & & $1 \cdot 32$ & $0.96,1 \cdot 81$ & 0.091 & & & \\
\hline Day 3-6 & $1 \cdot 26$ & $0.62,2.57$ & 0.523 & & & & 0.91 & $0.50,1.67$ & 0.771 & & & \\
\hline Day 7 or later & $1 \cdot 23$ & $0 \cdot 32,4 \cdot 71$ & $0 \cdot 759$ & & & & $2 \cdot 43$ & $0.84,7 \cdot 04$ & $0 \cdot 101$ & & & \\
\hline \multicolumn{13}{|l|}{ Household-level factors } \\
\hline \multicolumn{13}{|l|}{ Household wealth index } \\
\hline Poorest & 1.00 & & & & & & $1 \cdot 00$ & & & 1.00 & & \\
\hline Poorer & 0.86 & $0 \cdot 62,1 \cdot 21$ & $0 \cdot 386$ & & & & $1 \cdot 71$ & $1 \cdot 25,2 \cdot 35$ & 0.001 & $1 \cdot 65$ & $1 \cdot 20,2 \cdot 28$ & 0.002 \\
\hline Middle & 0.82 & $0 \cdot 59,1 \cdot 13$ & $0 \cdot 218$ & & & & $2 \cdot 04$ & $1 \cdot 47,2 \cdot 84$ & $<0.001$ & 1.55 & $1 \cdot 09,2 \cdot 22$ & 0.015 \\
\hline Richer & 0.72 & $0.51,1.01$ & 0.057 & & & & $2 \cdot 82$ & $2 \cdot 05,3 \cdot 88$ & $<0.001$ & $1 \cdot 78$ & $1 \cdot 26,2 \cdot 51$ & 0.001 \\
\hline Richest & 0.84 & $0.58,1 \cdot 22$ & 0.359 & & & & $4 \cdot 53$ & $3 \cdot 26,6 \cdot 30$ & $<0.001$ & $2 \cdot 41$ & $1 \cdot 62,3 \cdot 58$ & $<0.001$ \\
\hline \multicolumn{13}{|l|}{ Community-level factors } \\
\hline \multicolumn{13}{|l|}{ Residence } \\
\hline Rural & 1.00 & & & & & & $1 \cdot 00$ & & & & & \\
\hline Urban & 1.03 & $0 \cdot 81,1 \cdot 31$ & $0 \cdot 807$ & & & & $1 \cdot 71$ & $1 \cdot 39,2 \cdot 10$ & $<0.001$ & & & \\
\hline \multicolumn{13}{|l|}{ Geographical region (province) } \\
\hline Punjab & 1.00 & & & $1 \cdot 00$ & & & 1.00 & & & $1 \cdot 00$ & & \\
\hline Sindh & 1.91 & $1 \cdot 43,2 \cdot 54$ & $<0.001$ & $1 \cdot 88$ & $1 \cdot 40,2 \cdot 51$ & $<0.001$ & 0.58 & $0.46,0.73$ & $<0.001$ & 0.60 & $0 \cdot 48,0 \cdot 76$ & $<0.001$ \\
\hline North West Frontier & 0.79 & $0.57,1.08$ & 0.133 & 0.86 & $0 \cdot 62,1 \cdot 18$ & 0.347 & 0.47 & $0.35,0.63$ & $<0.001$ & 0.55 & $0.40,0.75$ & $<0.001$ \\
\hline Balochistan & 0.64 & $0.39,1.07$ & 0.087 & 0.68 & $0 \cdot 41,1 \cdot 14$ & $0 \cdot 143$ & 0.38 & $0.26,0.54$ & $<0.001$ & 0.47 & $0.31,0.71$ & $<0.001$ \\
\hline
\end{tabular}

*Divorced, separated and widowed. 


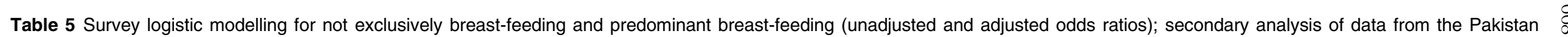
Demographic and Health Survey (PDHS) 2006-2007

\begin{tabular}{|c|c|c|c|c|c|c|c|c|c|c|c|c|}
\hline \multirow[b]{3}{*}{ Characteristic } & \multicolumn{6}{|c|}{ Not exclusively breast-feeding ( $<6$ months) } & \multicolumn{6}{|c|}{ Predominant breast-feeding ( $<6$ months $)^{\star}$} \\
\hline & \multicolumn{3}{|c|}{ Unadjusted } & \multicolumn{3}{|c|}{ Adjusted } & \multicolumn{3}{|c|}{ Unadjusted } & \multicolumn{3}{|c|}{ Adjusted } \\
\hline & OR & $95 \% \mathrm{Cl}$ & $P$ & OR & $95 \% \mathrm{Cl}$ & $P$ & OR & $95 \% \mathrm{Cl}$ & $P$ & OR & $95 \% \mathrm{Cl}$ & $P$ \\
\hline \multicolumn{13}{|l|}{ Individual-level factors } \\
\hline \multicolumn{13}{|l|}{ Maternal working status } \\
\hline Non-working & $1 \cdot 00$ & & & $1 \cdot 00$ & & & $1 \cdot 00$ & & & & & \\
\hline \multirow{2}{*}{\multicolumn{13}{|c|}{ Marital status }} \\
\hline & & & & & & & & & & & & \\
\hline \multicolumn{13}{|l|}{$\begin{array}{l}\text { Currently married } \\
\text { Formerly marriedt }\end{array}$} \\
\hline \multicolumn{13}{|l|}{ Maternal education } \\
\hline No education & $1 \cdot 00$ & & & & & & $1 \cdot 00$ & & & & & \\
\hline Primary & $1 \cdot 47$ & $0.92,2 \cdot 34$ & $0 \cdot 109$ & & & & 0.65 & $0.34,1 \cdot 22$ & $0 \cdot 187$ & & & \\
\hline Secondary or above & $1 \cdot 29$ & $0.91,1 \cdot 83$ & $0 \cdot 152$ & & & & 0.56 & $0.30,1.04$ & 0.068 & & & \\
\hline \multicolumn{13}{|l|}{ Literacy } \\
\hline Cannot read at all & $1 \cdot 00$ & & & $1 \cdot 00$ & & & 1.00 & & & $1 \cdot 00$ & & \\
\hline Able to read only passage & 0.92 & $0 \cdot 67,1 \cdot 28$ & 0.638 & 0.64 & $0.43,0.96$ & 0.029 & 0.57 & $0.35,0.92$ & 0.024 & 0.58 & $0.35,0.95$ & 0.033 \\
\hline \multicolumn{13}{|l|}{ Partner's education } \\
\hline No education & $1 \cdot 00$ & & & & & & 1.00 & & & & & \\
\hline Primary & 0.99 & $0.64,1.53$ & 0.955 & & & & $1 \cdot 58$ & $0 \cdot 87,2 \cdot 86$ & 0.125 & & & \\
\hline Secondary or above & $1 \cdot 06$ & $0 \cdot 70,1 \cdot 59$ & $0 \cdot 789$ & & & & $1 \cdot 04$ & $0 \cdot 65,1 \cdot 64$ & $0 \cdot 862$ & & & \\
\hline \multicolumn{13}{|l|}{ Mother’s age (years) } \\
\hline $15-19$ & $1 \cdot 00$ & & & & & & $1 \cdot 00$ & & & & & \\
\hline $20-34$ & 0.89 & $0.53,1.50$ & 0.658 & & & & 0.76 & $0 \cdot 38,1 \cdot 49$ & 0.427 & & & \\
\hline $35-49$ & 0.87 & $0.46,1.66$ & 0.674 & & & & 0.65 & $0.27,1.53$ & 0.326 & & & \\
\hline \multicolumn{13}{|l|}{ Birth order } \\
\hline First-born & $1 \cdot 00$ & & & & & & $1 \cdot 00$ & & & & & \\
\hline Second- to fourth-born & $1 \cdot 00$ & $0.67,1.51$ & 0.983 & & & & 0.84 & $0.50,1.41$ & 0.524 & & & \\
\hline Fifth-born or higher & 0.66 & $0.42,1.04$ & 0.070 & & & & 0.46 & $0.24,0.85$ & 0.013 & & & \\
\hline \multicolumn{13}{|l|}{ Preceding birth interval } \\
\hline No previous birth & $1 \cdot 00$ & & & & & & $1 \cdot 00$ & & & & & \\
\hline $0-14$ months & $1 \cdot 78$ & $0 \cdot 85,3 \cdot 73$ & $0 \cdot 127$ & & & & $1 \cdot 35$ & $0.53,3.42$ & 0.522 & & & \\
\hline 14-24 months & $1 \cdot 16$ & $0.69,1.94$ & 0.572 & & & & $1 \cdot 01$ & $0.53,1.91$ & 0.973 & & & \\
\hline$\geq 25$ months & 0.72 & $0.48,1.08$ & $0 \cdot 116$ & & & & 0.55 & $0.33,0.93$ & 0.028 & & & \\
\hline \multicolumn{13}{|l|}{ Sex of baby } \\
\hline Male & $1 \cdot 00$ & & & & & & $1 \cdot 00$ & & & & & \\
\hline Female & $1 \cdot 18$ & $0.87,1.60$ & 0.276 & & & & $1 \cdot 15$ & $0 \cdot 74,1 \cdot 78$ & 0.524 & & & \\
\hline Age of child (months) & $1 \cdot 42$ & $1 \cdot 29,1 \cdot 57$ & $<0.001$ & $1 \cdot 46$ & $1 \cdot 32,1 \cdot 62$ & $<0.001$ & $1 \cdot 42$ & $1 \cdot 24,1 \cdot 63$ & $<0.001$ & & & \\
\hline \multicolumn{13}{|l|}{ Perceived birth size of baby } \\
\hline Average & $1 \cdot 00$ & & & & & & $1 \cdot 00$ & & & & & \\
\hline Large & $1 \cdot 09$ & $0 \cdot 70,1 \cdot 69$ & $0 \cdot 713$ & & & & $1 \cdot 28$ & $0 \cdot 77,2 \cdot 13$ & 0.339 & & & \\
\hline Small & 1.06 & $0 \cdot 74,1 \cdot 52$ & $0 \cdot 758$ & & & & 0.35 & $0 \cdot 74,2 \cdot 46$ & $0 \cdot 314$ & & & \\
\hline Place of delivery & & & & & & & & & & & & \\
\hline Home & $1 \cdot 00$ & & & & & & $1 \cdot 00$ & & & & & \\
\hline Health facility & $1 \cdot 05$ & $0 \cdot 75,1 \cdot 46$ & 0.784 & & & & 0.56 & $0.35,0.90$ & 0.019 & & & \\
\hline Mode of delivery & & & & & & & & & & & & \\
\hline Non-Caesarean & $1 \cdot 00$ & & & & & & $1 \cdot 00$ & & & & & \\
\hline Caesarean section & $1 \cdot 10$ & $0.60,1.99$ & $0 \cdot 763$ & & & & 0.28 & $0.08,0.94$ & 0.039 & & & \\
\hline
\end{tabular}


Table 5 Continued

\begin{tabular}{|c|c|c|c|c|c|c|c|c|c|c|c|c|}
\hline \multirow[b]{3}{*}{ Characteristic } & \multicolumn{6}{|c|}{ Not exclusively breast-feeding ( $<6$ months) } & \multicolumn{6}{|c|}{ Predominant breast-feeding $(<6 \text { months })^{*}$} \\
\hline & \multicolumn{3}{|c|}{ Unadjusted } & \multicolumn{3}{|c|}{ Adjusted } & \multicolumn{3}{|c|}{ Unadjusted } & \multicolumn{3}{|c|}{ Adjusted } \\
\hline & OR & $95 \% \mathrm{Cl}$ & $P$ & OR & $95 \% \mathrm{Cl}$ & $P$ & OR & $95 \% \mathrm{Cl}$ & $P$ & OR & $95 \% \mathrm{Cl}$ & $P$ \\
\hline \multicolumn{13}{|l|}{ Type of delivery assistance } \\
\hline Health professional & $1 \cdot 00$ & & & & & & $1 \cdot 00$ & & & $1 \cdot 00$ & & \\
\hline Traditional birth attendant & $1 \cdot 23$ & $0 \cdot 85,1 \cdot 78$ & $0 \cdot 282$ & & & & $2 \cdot 28$ & $1 \cdot 39,3 \cdot 74$ & 0.001 & $1 \cdot 96$ & $1 \cdot 18,3 \cdot 24$ & 0.009 \\
\hline Other & $0 \cdot 86$ & $0.58,1.26$ & 0.429 & & & & $1 \cdot 37$ & $0 \cdot 79,2 \cdot 38$ & 0.255 & $1 \cdot 33$ & $0 \cdot 75,2 \cdot 34$ & $0 \cdot 324$ \\
\hline \multicolumn{13}{|l|}{ Antenatal clinic visits } \\
\hline None & $1 \cdot 00$ & & & & & & $1 \cdot 00$ & & & & & \\
\hline $1-3$ & $1 \cdot 14$ & $0 \cdot 82,1 \cdot 60$ & 0.434 & & & & 0.94 & $0.58,1.51$ & $0 \cdot 814$ & & & \\
\hline$\geq 4$ & $1 \cdot 36$ & $0.92,2 \cdot 00$ & $0 \cdot 128$ & & & & $0 \cdot 84$ & $0.48,1.47$ & 0.556 & & & \\
\hline \multicolumn{13}{|l|}{ Timing of postnatal check-up } \\
\hline Immediate (hospital birth) & $1 \cdot 00$ & & & & & & $1 \cdot 00$ & & & & & \\
\hline Day 0-2 & $1 \cdot 40$ & $0 \cdot 80,2 \cdot 45$ & 0.235 & & & & $1 \cdot 11$ & $0 \cdot 49,2 \cdot 50$ & $0 \cdot 791$ & & & \\
\hline Days 3-6 & $1 \cdot 32$ & $0.50,3.50$ & 0.580 & & & & $1 \cdot 24$ & $0 \cdot 31,4.90$ & 0.752 & & & \\
\hline Day 7 or later & $1 \cdot 64$ & $0.26,9.53$ & 0.600 & & & & $3 \cdot 40$ & $0 \cdot 26,43 \cdot 6$ & 0.344 & & & \\
\hline \multicolumn{13}{|l|}{ Household-level factors } \\
\hline \multicolumn{13}{|l|}{ Household wealth index } \\
\hline Poorest & $1 \cdot 00$ & & & $1 \cdot 00$ & & & $1 \cdot 00$ & & & & & \\
\hline Poorer & $0 \cdot 80$ & $0.51,1 \cdot 26$ & 0.336 & 0.98 & $0.61,1.58$ & 0.943 & 0.68 & $0 \cdot 39,1 \cdot 18$ & $0 \cdot 175$ & & & \\
\hline Middle & 0.94 & $0.57,1.55$ & 0.817 & 1.07 & $0 \cdot 62,1 \cdot 86$ & 0.803 & 0.38 & $0 \cdot 19,0.77$ & 0.007 & & & \\
\hline Richer & $1 \cdot 16$ & $0 \cdot 71,1 \cdot 91$ & 0.550 & $1 \cdot 69$ & $0.95,3.00$ & 0.072 & $0 \cdot 73$ & $0 \cdot 38,1 \cdot 38$ & $1 \cdot 389$ & & & \\
\hline Richest & 1.54 & $0.92,2.56$ & $0 \cdot 101$ & $2 \cdot 31$ & $1 \cdot 22,4 \cdot 36$ & $0 \cdot 010$ & $0 \cdot 61$ & $0 \cdot 29,1 \cdot 24$ & $1 \cdot 246$ & & & \\
\hline \multicolumn{13}{|l|}{ Community-level factors } \\
\hline \multicolumn{13}{|l|}{ Residence } \\
\hline Rural & $1 \cdot 00$ & & & & & & $1 \cdot 00$ & & & & & \\
\hline Urban & 1.09 & $0.76,1.56$ & 0.640 & & & & $1 \cdot 49$ & $0.88,2.52$ & $0 \cdot 133$ & & & \\
\hline \multicolumn{13}{|l|}{ Geographical region (province) } \\
\hline Punjab & $1 \cdot 00$ & & & $1 \cdot 00$ & & & 1.00 & & & $1 \cdot 00$ & & \\
\hline Sindh & 0.81 & $0.56,1.19$ & 0.285 & $0 \cdot 77$ & $0.51,1 \cdot 16$ & 0.204 & $0 \cdot 77$ & $0.46,1.28$ & 0.324 & $0 \cdot 78$ & $0.47,1 \cdot 28$ & 0.331 \\
\hline North West Frontier & 0.39 & $0.26,0.60$ & $<0.001$ & $0 \cdot 37$ & $0.23,0.59$ & $<0.001$ & 0.55 & $0.30,1.01$ & 0.055 & 0.54 & $0.29,1.00$ & 0.052 \\
\hline Balochistan & $0 \cdot 87$ & $0.49,1.54$ & 0.637 & 0.69 & $0 \cdot 37,1 \cdot 28$ & 0.240 & 0.65 & $0 \cdot 20,2 \cdot 12$ & $0 \cdot 480$ & 0.58 & $0 \cdot 17,1 \cdot 96$ & 0.384 \\
\hline
\end{tabular}

*Logistic model based on proportions of predominant $(n$ 179) and exclusive breast-feeding ( $n$ 354).

tDivorced, separated and widowed. 
had better contact with the health-care delivery system were less likely to practise EBF. This trend was seen in Nepal too, where increased antenatal visits had a negative effect on EBF; while in India more ANC visits had a positive effect on $\mathrm{EBF}^{(21,22)}$. In India women who were delivered at a health-care facility had a lower EBF rate whereas in Sri Lanka increased postnatal contacts with the health system improved the EBF rate ${ }^{(22,23)}$. The trend in Pakistan could be due to a lack of training of health-care workers in breast-feeding counselling.

Unfortunately, the attractive marketing strategies of manufacturers of formula milk as substitutes of mother's milk are not in line with the Legislation Ordinance on Breastfeeding issued by Government of Pakistan in $2002^{(24)}$; this was also shown in a study from Karachi where the majority $(P<0 \cdot 0001)$ of doctors supported the idea of receiving gifts, promotional material and donations from the formula milk/feeding bottle manufacturers ${ }^{(25)}$. According to the study in Karachi more than 95\% of mothers considered that breast milk was the best food for their infant, but the practices of urban mothers were quite contradictory to this statement showing a gap between knowledge and practices. According to WHO, Iran was the first among Eastern Mediterranean countries to approve the WHO's international code of marketing on breast milk substitutes, adopt it as national law and implement all aspects of the code ${ }^{(26)}$, which explains the higher EBF and lower bottle-feeding rates in Iran compared with Pakistan. There is need to develop and implement guidelines for all levels of health-care workers on how to counsel mothers about the importance of EBF. The undergraduate medical syllabus in Pakistan needs revision and should include an essential standardized infant and young child feeding (IYCF) training package. It is important to revive the Baby Friendly Hospital Initiative (BFHI) to ensure that all children born in hospitals are put to the breast within the first hour of delivery, the rate of which is low (27.3\%) in Pakistan. All staff providing mother and child health services must also receive training in counselling about young infant feeding and nutrition. A system of close monitoring and evaluation should be put in place for the continuing accreditation of health facilities offering obstetric and child health services. This should also include the institutions operating in the private health sector. Furthermore, EBF rates in Pakistan could also be increased through community health workers (lady health workers) ${ }^{(27)}$ by providing counselling to women during antenatal, natal and postnatal home visits, as the majority of deliveries in Pakistan take place at home. A clinical trial has demonstrated a positive effect of home visiting by registered midwives on improving EBF rate ${ }^{(28)}$.

Achieving increased frequency of EBF requires behaviour change in mothers and other members of the family who influence decision making. Accomplishing behaviour change could be a formidable target, as multiple behaviour changes might be required in mothers who are bottle-feeding or non-EBF. On the other hand, a single behaviour change which aims at convincing 'at risk' women not to give water and other non-nutritious liquids may be a feasible achievement. Demographic and Health Survey data from Pakistan show that the profile of mothers who predominantly breast-feed and those who non-exclusively breast-feed are similar, as both are being practised more by illiterate mothers. This finding has programmatic implications. Since the target population for a behaviour change campaign is same it might be easier to develop public health messages which effectively highlight the disadvantages of giving water and waterbased liquids before 6 months of age. This would have the potential of significantly increasing the rate of EBF with its attendant benefits. Such changes have been achieved by programmes in Cambodia ${ }^{(29,30)}$ where the EBF rates were increased from $11 \%$ to $60 \%$ in five years by targeting mothers practising PBF. Similar programmes in Zambia and Ghana have also produced positive changes in the behaviour of mothers, thereby increasing EBF rates ${ }^{(31)}$.

Another factor having an adverse association with EBF was urban residence as these women were more likely to bottle-feed their babies, a finding which is similar to Sri Lanka ${ }^{(23)}$. In Bangladesh urban mothers did not initiate timely breast-feeding for their infants ${ }^{(32)}$. This paradox could be utilized to the advantage of breast-feeding advocacy programmes, since urban mothers have a much better access to various sources of information that can be used to create awareness regarding the benefits of breast-feeding.

The present secondary analysis also highlighted significantly lower rates of EBF among working mothers. The current legislation in Pakistan for maternity leave and infrastructure to facilitate working women in breastfeeding is not supportive of EBF practices ${ }^{(24)}$. Most workplaces neither have space nor any system of support in place for mothers who want to breast-feed at their place of work. Studies from Iran show that giving a $1 \mathrm{~h}$ break daily to mothers for breast-feeding and also facilitating them in the practice have increased the EBF rates ${ }^{(33)}$. National paediatric associations and other similar organizations have an important role as pressure groups to influence parliamentarians to legislate for breast-feeding friendly policies in workplaces. Efforts must be made to ensure there is a breast-feeding friendly environment in both public and private sector workplaces.

The large geographic variation in EBF, with low rates in Punjab Province and high rates in North West Frontier Province, may need further study as the current analysis did not allow us to explore the behavioural, environmental and social factors affecting mothers' decisions regarding infant feeding practices. Some common factors such as changing lifestyles, aggressive promotion of formula milk, a general unawareness of the importance of breast-feeding and perceived insufficiency of milk could play important roles in contributing towards suboptimal 
feeding practices. In women delivered through Caesarean section there is a perceived insufficiency of milk contributing towards high bottle-feeding rate, which is a common finding in India, Nepal, Sri Lanka and China where it was the main reason for delayed contact ${ }^{(21,34-36)}$. This perceived insufficiency was also found to be the most common cause for discontinuing breast-feeding of children in Australia ${ }^{(37)}$.

The main strengths of our study are the nationally representative sample, the comprehensive data on standard infant feeding indicators and the appropriate adjustments for sampling design made in the analysis. However, the major limitation is the $24 \mathrm{~h}$ recall method used for estimating EBF rates in the current survey. Although this method is recommended by WHO, there is a concern that it considerably overestimates EBF rates as it excludes children who were given liquids and foods at infrequent intervals $^{(38-43)}$. In Sri Lanka the EBF rate was reported as $77 \cdot 4 \%$ by using ' $24 \mathrm{~h}$ recall' and as $49 \cdot 1 \%$ by another method of 'recall since birth' ${ }^{\text {(44) }}$. Therefore, it is quite possible that the EBF rates in Pakistan might be even lower than the levels estimated in the current analysis.

\section{Conclusions}

The EBF rates in Pakistan are the lowest and the bottlefeeding rates are the highest in South Asia. This should be considered as a wakeup call for child health programmes to focus more on improved infant feeding practices, especially EBF. There is an urgent need to involve all national programmes delivering child health interventions at all levels of health-care infrastructure. There should be enhanced training of health-care providers in infant and young child nutritional counselling. There is a need for breast-feeding friendly laws and their effective implementation to improve the rates of EBF in Pakistan. The advocacy campaigns should focus on all women of childbearing age at all levels of society, with special emphasis on educated, working women from upper socio-economic strata.

\section{Acknowledgements}

The study was supported by AusAID through the Public Sector Linkages Program. No author has any conflict of interest on the content of this manuscript. T.H., Y.B.N., N.K. and S.A. designed the study, obtained data sets, guided analysis and wrote the manuscript. T.H., Y.B.N., N.K., D.-S.A. and A.M.K. obtained the literature, checked results and reviewed and revised the manuscript. K.E.A. wrote the results section and interpreted results. K.E.A. and S.A. converted data files, conducted statistical analyses and compiled the results tables. M.J.D. conceptualized the research question, designed and guided the analysis and edited the manuscript. The National Institute of Population
Studies of Pakistan carried out the PDHS 2006-2007 for the Health Sector Development Project of the Ministry of Health. A fellowship sponsored by the Australian Leadership Awards (ALA) scheme and a workshop funded by the Public Sector Linkages Program (PSLP) of the AusAID facilitated the data analysis and writing. Dr Upal Senarath, Principal Investigator SAIFRN Sri Lanka Chapter, provided the technical support.

\section{References}

1. Dewey K, Heinig MJ \& Nommsen-Rivers L (1995) Differences in morbidity between breast-fed and formulafed infants. J Pediatr 126, 696-702.

2. Leung A \& Sauve R (2005) Breast is best for babies. J Natl Med Assoc 97, 1010-1019.

3. Wright AL, Holberg CJ, Martinez FD et al. (1989) Breast feeding and lower respiratory tract illness in the first year of life. Group Health Medical Associates. BMJ 299, 946-949.

4. Bahl R, Frost C, Kirkwood BR et al. (2005) Infant feeding patterns and risks of death and hospitalization in the first half of infancy: multicentre cohort study. Bull World Health Organ 83, 418-426.

5. Howie PW, Forsyth JS, Ogston SA et al. (1990) Protective effect of breast feeding against infection. BMJ 300, 11-16.

6. Arifeen S, Black RE, Antelman G et al. (2001) Exclusive breastfeeding reduces acute respiratory infection and diarrhea deaths among infants in Dhaka slums. Pediatrics 108, E67.

7. World Health Organization (2002) Infant and Young Child Nutrition; Global Strategy on Infant and Young Child Feeding. Resolution WHA55/15. Geneva: WHO.

8. Black RE, Allen LH, Bhutta ZA et al. (2008) Maternal and child undernutrition: global and regional exposures and health consequences. Lancet 371, 243-260.

9. Jones G, Steketee RW, Black RE et al. (2003) How many child deaths can we prevent this year? Lancet 362, 65-71.

10. World Health Organization (2002) Infant and Young Child Nutrition; Global Strategy on Infant and Young Child Feeding. Report no. A55/15. Geneva: WHO.

11. Oddy WH, Sly PD, de Klerk NH et al. (2003) Breast feeding and respiratory morbidity in infancy: a birth cohort study. Arch Dis Child 88, 224-228.

12. Temboury MC, Otero A, Polanco I et al. (1994) Influence of breast-feeding on the infant's intellectual development. J Pediatr Gastroenterol Nutr 18, 32-36.

13. Government of Pakistan (2005) Pakistan Economic Survey. Islamabad: Government of Pakistan.

14. National Institute of Population Studies \& Macro International Inc. (2008) Pakistan Demographic and Health Survey 2006-07. Islamabad/Calverton, MD: NIPS/Macro International Inc.

15. World Health Organization (2008) Indicators for Assessing Infant and Young Child Feeding Practices. Washington, DC: WHO.

16. Filmer D \& Pritchett LH (2001) Estimating wealth effects without expenditure data - or tears: an application to educational enrolments in states of India. Demography 38, 115-132.

17. Department of Census and Statistics Sri Lanka (2000) Sri Lanka Demographic and Health Survey 2000. Colombo: Department of Census and Statistics Sri Lanka.

18. National Institute of Population Research and Training, Mitra and Associates \& ORC Macro (2001) Bangladesh Demographic and Health Survey 2000. Dhaka/Calverton, MD: National Institute of Population Research and Training, Mitra and Associates/ORC Macro. 
19. International Institute for Population Sciences \& ORC Macro (2000) National Family Health Survey (NFHS-2), 1998-99, India. Mumbai: IIPS.

20. Ministry of Health Nepal, New ERA \& ORC Macro (2002) Nepal Demographic and Health Survey 2001. Kathmandu/ Calverton, MD: Family Health Division, Ministry of Health, New ERA/ORC Macro.

21. Pandey S, Tiwari K, Senarath U et al. (2010) Determinants of infant and young child feeding practices in Nepal: secondary data analysis of Demographic and Health Survey 2006. Food Nutr Bull 31, 334-351.

22. Patel A, Badhoniya N, Khadse $S$ et al. (2010) Infant and young child feeding indicators and determinants of poor feeding practices in India: secondary data analysis of National Family Health Survey 2005-06. Food Nutr Bull 31, 314-333.

23. Senarath U, Dibley MJ, Godakandage SS et al. (2010) Determinants of infant and young child feeding practices in Sri Lanka: secondary data analysis of Demographic and Health Survey 2000. Food Nutr Bull 31, 352-365.

24. Government of Pakistan (2002) The Protection of Breast Feeding and Child Nutrition Ordinance. The Gazette of Pakistan. http://www.thenetwork.org.pk/Resources/Legislations/ PDF/12-8-2011-0-51-50-603-Legislation\%20ordinance\%20on \%20breastfeeding.pdf (accessed May 2011).

25. Hanif R, Khalil E, Sheikh A et al. (2008) Knowledge about breastfeeding in accordance with the national policy among doctors, paramedics and mothers in baby-friendly hospitals. J Pak Med Assoc 60, 881-886.

26. World Health Organization (2006) Breast feeding breakthroughs. http:/www.unicef.org/sowc98/panel13.htm (accessed January 2011).

27. Nisar YB (2008) Corrections needed to Pakistani programme details. Bull World Health Organ 86, B.

28. Bashour HN, Kharouf MH, Abdul Salam AA et al. (2008) Effect of postnatal home visits on maternal/infant outcomes in Syria: a randomized controlled trial. Public Health Nurs 25, 115-125.

29. Anonymous (2002) Cambodia 2000: results from the Demographic and Health Survey. Stud Fam Plann 33, 269-273.

30. Islam MM, Khatun M, Peerson JM et al. (2008) Effects of energy density and feeding frequency of complementary foods on total daily energy intakes and consumption of breast milk by healthy breastfed Bangladeshi children. Am J Clin Nutr 88, 84-94.

31. Mangasaryan N (2010) Effective Strategies to Improve Infant and Young Child Feeding. Managua: UNICEF.

32. Mihrshahi S, Kabir I, Roy SK et al. (2010) Determinants of infant and young child feeding practices in Bangladesh: secondary data analysis of Demographic and Health Survey 2004. Food Nutr Bull 31, 295-313.

33. Zareai M, O'Brien ML \& Fallon AB (2007) Creating a breastfeeding culture: a comparison of breastfeeding practises in Australia and Iran. Breastfeed Rev 15, 15-20.

34. Patel A, Badhoniya N, Khadse S et al. (2010) Infant and young child feeding indicators and determinants of poor feeding practices in India: secondary data analysis of National Family Health Survey 2005-06. Food Nutr Bull 31, 314-333.

35. Senarath U, Siriwardena I, Godakandage SS et al. (2012) Determinants of breastfeeding practices: an analysis of the Sri Lanka Demographic and Health Survey 2006-2007. Matern Child Nutr 8, 315-329.

36. Zhao Y, Niu AM, Xu GF et al. (2003) Early infant feeding practices in Jinan City, Shandong Province, China. Asia Pac J Clin Nutr 12, 104-108.

37. Australian Bureau of Statistics (2003) Breastfeeding in Australia. http://www.abs.gov.au/ausstats/abs@.nsf/mf/ 4810.0.55.001 (accessed May 2011).

38. Webb K, Marks GC, Lund-Adams M et al. (2001) Towards A National System For Monitoring Breastfeeding in Australia: Recommendations For Population Indicators, Definitions and Next Steps. Canberra: Commonwealth of Australia.

39. Piwoz EG, Creed de Kanashiro H, Lopez de Romana G et al. (1995) Potential for misclassification of infants' usual feeding practices using 24-hour dietary assessment methods. J Nutr 125, 57-65.

40. Zohoori N, Popkin BM \& Fernandez ME (1993) Breastfeeding patterns in the Philippines: a prospective analysis. J Biosoc Sci 25, 127-138.

41. Aarts C, Kylberg E, Hornell A et al. (2000) How exclusive is exclusive breastfeeding? A comparison of data since birth with current status data. Int J Epidemiol 29, 1041-1046.

42. Ssenyonga R, Muwonge R \& Nankya I (2004) Towards a better understanding of exclusive breastfeeding in the era of HIV/AIDS: a study of prevalence and factors associated with exclusive breastfeeding from birth, in Rakai, Uganda. $J$ Trop Pediatr 50, 348-353.

43. Engebretsen IM, Wamani H, Karamagi C et al. (2007) Low adherence to exclusive breastfeeding in Eastern Uganda: a community-based cross-sectional study comparing dietary recall since birth with 24-hour recall. $B M C$ Pediatr 7, 10.

44. Agampodi SB, Fernando S \& Dharmaratne SD (2009) Comparison of definitions used for exclusive breast feeding - the Labbock's strict definition and the WHO definition as methods of estimating exclusive breastfeeding rates in Sri Lanka. Ceylon Med J 54, 20. 